\title{
Effect of dignity therapy on end-of-life psychological distress in terminally ill Portuguese patients: A randomized controlled trial
}

\author{
MIGUEL JULIÃO, M.D., M.SC., PH.D., ${ }^{1}$ FÁTIMA OLIVEIRA, R.N., ${ }^{2}$ BALTAZAR NUNES, PH.D., ${ }^{3}$ \\ ANTÓNIO VAZ CARNEIRO, M.D., PH.D., ${ }^{4}$ AND ANTÓNIO BARBOSA, M.D., PH.D. ${ }^{5}$ \\ ${ }^{1}$ Life and Health Sciences Research Institute, School of Medicine, University of Minho, Braga, Portugal \\ ${ }^{2}$ Department of Palliative Medicine, Instituto das Irmãs Hospitaleiras do Sagrado Coração de Jesus, Casa de Saúde da \\ Idanha, Belas, Portugal \\ ${ }^{3}$ Department of Epidemiology, Instituto Dr. Ricardo Jorge, Lisbon, Portugal \\ ${ }^{4}$ Center for Evidence-Based Medicine, Faculty of Medicine of the University of Lisbon, Lisbon, Portugal \\ ${ }^{5}$ Center for Bioethics, Faculty of Medicine, University of Lisbon, Lisbon, Portugal
}

(RECEIVED March 18, 2016; ACCEPTED January 26, 2017)

\begin{abstract}
Objective: Dignity therapy (DT) is a brief form of psychotherapy developed for patients living with a life-limiting illness that has demonstrated efficacy in treating several dimensions of endof-life psychological distress. Our aim was to determine the influence of DT on demoralization syndrome (DS), the desire for death (DfD), and a sense of dignity (SoD) in terminally ill inpatients experiencing a high level of distress in a palliative care unit.

Method: A nonblinded phase II randomized controlled trial was conducted with 80 patients who were randomly assigned to one of two groups: the intervention group (DT + standard palliative care [SPC]) or the control group (SPC alone). The main outcomes were DS, DfD, and SoD, as measured according to DS criteria, the Desire for Death Rating Scale, and the Patient Dignity Inventory (PDI), respectively. All scales were assessed at baseline (day 1) and at day 4 of follow-up. This study is registered with http://www.controlled-trials.com/ISRCTN34354086.

Results: Of the 80 participants, 41 were randomized to DT and 39 to SPC. Baseline characteristics were similar between the two groups. DT was associated with a significant decrease in DS compared with SPC (DT DS prevalence $=12.1 \%$; SPC DS prevalence $=60.0 \%$; $p<0.001$ ). Similarly, DT was associated with a significant decrease in DfD prevalence (DT DfD prevalence $=0 \%$; SPC DfD prevalence $=14.3 \% ; p=0.054$ ). Compared with participants allocated to the control group, those who received DT showed a statistically significant reduction in 19 of 25 PDI items.

Significance of results: Dignity therapy had a beneficial effect on the psychological distress encountered by patients near the end of life. Our research suggests that DT is an important psychotherapeutic approach that should be included in clinical care programs, and it could help more patients to cope with their end-of-life experiences.
\end{abstract}

KEYWORDS: Dignity therapy, End-of-life psychological distress, Randomized controlled trial, Palliative care

Address correspondence and reprint requests to Miguel Julião, Life and Health Sciences Research Institute, School of Medicine, University of Minho, Braga, Portugal. E-mail: migueljuliao@ gmail.com.

\section{INTRODUCTION}

Patients with life-threatening illnesses face great psychological challenges and frequently experience a high prevalence of emotional distress, including depression and anxiety (Block, 2001). It is becoming increasingly clear that there are other significant 
forms and expressions of patient suffering toward the end of life (Block, 2000; Julião, 2014). This end-of-life psychological experience includes such constructs as demoralization syndrome (DS), the desire for death (DfD), and a loss of dignity (Julião, 2014).

Demoralization is a common psychiatric disorder associated with the end of life and has been commonly observed in the medically and psychiatrically ill. Hopelessness, helplessness, and the loss of purpose and meaning in life - the loss of morale-are key and central symptoms of DS. This psychological construct is associated with chronic medical illnesses, disabilities, bodily disfigurement, loss of dignity, social isolation, feelings of greater dependency on others, and/or the perception of being a burden (Kissane et al., 2001).

Various studies have addressed the issue of DfD and requests for euthanasia and physician-assisted suicide among terminally ill patients (Chochinov et al., 1995; Breitbart et al., 2000; Mystakidou et al., 2005). The evidence shows that DfD is associated with complex and multifactorial factors, and social and psychological variables, such as depression, pain, and physical functioning, as well as deterioration in quality of life and loss of dignity (Hudson et al, 2006).

Psychological suffering for terminally ill patients is also often framed in terms of loss of dignity (Chochinov, 2007). Dignity can be defined as the quality of being worthy of honor or respect. The concept of dignity was always one of the central aspects of medicine. To think about the modern concept of dignity in its many different aspects is an imperative of modern medicine. This concept has been an integral part of human life and culture since ancient times, integrating our life experiences, victories, and sufferings. However, despite being an inalienable aspect of every human being, something that belongs to every individual, dignity has also been somewhat foreign to our individual and collective consciousness. Over the last 20 years, there has been a significant scientific investment in the clarification and implementation within the field of medicine of the often vague and misused concept of dignity, especially in palliative medicine and through working with patients at the end of their lives (Julião, 2015). The empirical work by Chochinov and colleagues (2002) regarding the concept of dignity in terminally ill patients led to the creation of their Model of Dignity, which offered important insights into how patients face terminal illness in terms of illness-, social-, and personal-related factors, which might empower or undermine one's sense of dignity (SoD). This model was the basis for the creation of dignity therapy (DT), a brief individualized intervention designed to address psychosocial and existential distress among terminally ill patients (Chochinov et al., 2005).

Previous trials were designed to study DT's efficacy with respect to end-of-life distress compared with standard palliative care (SPC) and other interventions (Hall et al., 2011; Chochinov et al., 2011; Julião et al., 2013a; 2014). Chochinov and coworkers (2011) compared DT with SPC and a client-centered intervention and found that, although DT did not significantly reduce distress, those assigned to DT were significantly more likely than those in other study groups to find this approach helpful, to report improved quality of life, an increased SoD, and a change in how their family saw and appreciated them. While DT did not demonstrate any effect on depression and anxiety, the authors stated that this was likely due to the low baseline rates of distress. For this reason, Julião et al. (2013a; 2014) conducted a randomized controlled trial (RCT) that targeted more distressed patients. They found that DT outperformed SPC and significantly ameliorated both depression and anxiety.

In the RCT by Julião (2014), other psychosocial variables were studied, including DS, DfD, and SoD. The present paper reports the findings of our comparison of how DT and SPC affect DS, DfD, and $\mathrm{SoD}$ in a cohort of 80 terminally ill patients who were being cared for in a Portuguese palliative care unit.

\section{METHODS}

This phase II nonblinded RCT comprised two study arms: an intervention group (DT plus SPC) and a control group (SPC alone). Given the nature of the study design, it was not possible for the investigators or patients to be blinded to study arm assignment (http:// www.controlled-trials.com/ISRCTN34354086).

\section{Participants}

Recruitment took place from the S. Bento Menni tertiary inpatient palliative medicine unit in Lisbon over the course of 36 months (May of 2010 to May of 2013). The inclusion criteria were as follows: being $\geq 18$ years of age; having a life-threatening disease with a prognosis of 6 months or less; showing no evidence of dementia or delirium (as determined by chart review or clinical consensus); a Mini-Mental State score $\geq 20$; having the ability to read and speak Portuguese and provide written informed consent; and being available for four to five research encounters over the period of a month. The study was approved by the Ethics Committee of the Instituto das Irmãs Hospitaleiras do Sagrado Coração de Jesus 
and the Ethics Committee of the Faculty of Medicine at the University of Lisbon.

\section{Randomization and Masking}

The randomization process was computer-generated, using a fixed block of four, and was conducted by an independent statistician. The selection process did not take into account patients' background variables-namely, levels of psychological distress at baseline. Allocation concealment was achieved using sequentially numbered envelopes for consecutive trial participants. After baseline measures were obtained by the secondary investigator (a trained nurse, F.O.), the envelope was opened in the presence of the patient to reveal to which study arm they had been assigned. While the principal investigator (M.J.) was not blind to patients' arm assignments, he was blind to the psychometric results throughout the entire protocol. The secondary investigator was blind to the content of DT sessions.

\section{Interventions}

\section{Standard Palliative Care}

SPC was provided by a multiprofessional palliative care team comprised of three palliative care physicians (one of whom was M.J.), nurses, a psychologist, a social worker, and a spiritual assistant, thus being well poised to meet the physical, existential, and psychosocial needs of patients and families, meeting once daily, in patients' individual rooms. Clinical care included regular clinical follow-up: physical examination, symptom assessment and management, and clinical interviews (median $=25$ minutes; range $=10-30$ ). SPC was provided for all patients throughout their entire hospital stay, including the period of time they were enrolled in the RCT. Aside from not receiving DT, participants randomized to SPC were provided regular assessment and management by the entire multiprofessional team. Other than the principal and secondary investigators, the remaining members of the palliative care team were not informed as to which patients were enrolled in the study, or whether or not they received DT. This reduced any potential for bias or alteration of care based on knowledge of arm assignment. The principal and secondary investigators had limited clinical contact with patients, given that the former worked one day a week on the palliative care ward and the latter was the chief nurse and had little direct patient contact.

\section{Dignity Therapy}

DT is a brief psychotherapeutic intervention designed to bolster a patient's sense of meaning and purpose in order to reinforce a continued sense of worth, within a framework that is supportive, nurturing, and accessible. DT is based on an empirical model of dignity in the terminally ill (Chochinov et al., 2002). Various facets of the model have informed key elements of this therapeutic modality, likely accounting for its beneficial effects on patients and families. The creation of a legacy document reflects the generativity component of the model, while the questions focused on roles, accomplishments, meaning, and purpose - and the opportunity to share hopes, wishes, and life lessons with those they will soon leave behind-are based on core dignity model themes and subthemes (e.g., care tenor, continuity of self, role preservation, and acceptance). Patients enrolled in DT were guided by a trained therapist (M.J.) to respond to questions about the aspects of their lives they would most want their loved ones to know about or remember.

Therapeutic sessions, lasting between 30 and 60 minutes, were offered at the patient's bedside and audiotaped. Every tape-recorded session was erased after completion of the study protocol. Our study utilized the DT question framework (see Table 1) (Chochinov et al., 2005), as per previously published trials. All DT sessions were conducted by the principal investigator (M.J.), who attended an international DT workshop held in Canada hosted by the developers of DT. All DT procedures were conducted in accordance with those described and published

\section{Table 1. Dignity therapy question protocol}

Tell me a little about your life history, particularly the parts that you either remember most or think are the most important? When did you feel most alive?

Are there particular things that you would want your family to know about you, and are there particular things you would want them to remember?

What are the most important roles you have played in your life (family roles, vocational roles, community service roles, etc.)? Why were they so important to you, and what do you think you accomplished within those roles?

What are your most important accomplishments, and what do you feel most proud of?

Are there particular things that you feel still need to be said to your loved ones, or things that you would want to take the time to say once again?

What are your hopes and dreams for your loved ones?

What have you learned about life that you would want to pass along to others? What advice or words of guidance would you wish to pass along to your [son, daughter, husband, wife, parents, other(s)]?

Are there words or perhaps even instructions you would like to offer your family in order to provide them with comfort or solace?

In creating this permanent record, are there other things that you would like included? 
by Chochinov et al. (2005; 2011). A senior psychiatrist (A.B.) acquainted with DT vetted randomly selected DT transcripts in order to monitor fidelity and adherence to the protocol.

Patients meeting the eligibility criteria and agreeing to participate in the study were asked to provide written informed consent. Once patients completed the baseline assessment, those randomized to DT were provided with the standard framework of questions, thus giving them time to reflect upon and shape their eventual responses. The taped DT session was scheduled to take place within two or three days. The therapeutic session was guided by the DT framework. Once a taped session was complete, the patient's recorded dialogue was transcribed verbatim and then edited and reshaped into a written narrative over the course of the next two to three days. The editing process was performed entirely by the therapist (M.J.) and consisted of eliminating colloquialisms, nonstarters, and interruptions, and also correcting chronological errors. Once this editing process was complete, another session was arranged as soon as possible where the therapist could read the document to the patient, allowing for final editorial corrections and revisions. The final version of the generativity document was given to the patient, to be distributed to individuals of their choosing.

\section{Measures}

The present paper reports on secondary outcomes: DS, DfD, and SoD. The categorical and clinician rating diagnostic criteria for DS include all of the following items: (1) an experience of emotional distress, such as hopelessness or having lost meaning and purpose in life; (2) attitudes of helplessness, failure, and pessimism, and a loss of faith in a worthwhile future; (3) a reduced ability to cope and respond flexibly; (4) social isolation and deficient social support; and (5) persistence of the abovementioned phenomena for two or more weeks (Kissane et al., 2001). Kissane and colleagues did not report any data on validity and reliability. All five criteria had to be present in order to diagnose DS and then study its prevalence.

In 1995, Chochinov et al. developed a global clinical tool to rate patients' DfD called the Desire for Death Rating Scale (DDRS), but no data regarding its reliability or validity were reported. Using openended questions, the DDRS allows for clinician ratings of DfD on a scale of 0 to 6 . A score below 3 means that a patient has no DfD, while patients with a serious or pervasive DfD had scores $\geq 4$. To identify patients with DfD and its prevalence, we used a cutoff score of $\geq 4$ on the DDRS. The PDI (Chochinov et al., 2008) was employed to assess SoD. This is a 25-item scale developed to provide a measure of dignity-related distress and serve as a screening tool to assess a broad range of issues that have been reported to influence one's sense of dignity. Each item is rated on a 5-point scale, where $1=$ not a problem, $2=$ a slight problem, $3=$ a problem, $4=a$ major problem, and $5=$ an overwhelming problem. The value of Cronbach's $\alpha$ for the PDI was 0.93 , and its test-retest reliability was $r=0.85$. Evidence for concurrent validity was reported by way of significant associations between PDI factors and concurrent measures of distress (Chochinov et al., 2008).

All the measurements taken in our study were assessed at baseline (T1) and at day 4 (T2) post-DT, as per the study protocol.

\section{Statistical Analysis}

We compared continuous variables and ordinal rating scales using Mann-Whitney nonparametric statistical tests, while Fisher's exact test was employed for independent group comparisons (DT vs. SPC), and McNemar's test was utilized for paired comparisons between each timepoint ( $\mathrm{T} 2$ and baseline) within each study group. The criterion for statistical significance was set at $p<0.05$ in a two-tailed test. The statistical analysis was applied to all patients who had at least one complete evaluation at any given follow-up point.

\section{RESULTS}

The summary demographic and illness data for both groups are presented in Table 2 . There were no differences between the two groups on baseline characteristics. Over a 36-month period, 150 patients were admitted to the palliative care ward. A total of 92 were assessed for eligibility, 80 of whom were randomized (41 to DT and 39 to SPC). Some 12 patients were not randomized as a result of not meeting the inclusion criteria $(n=8)$ and declining to participate $(n=4)$. After randomization, one patient died before receiving DT and another clinically deteriorated rapidly; 10 patients were lost to follow-up (as a result of death, clinical deterioration, or declining further participation) at T2 (DT $n=6 ; \mathrm{SPC} n=4)$ (see the CONSORT diagram reported elsewhere) (Julião et al., $2013 a$; 2014). The mean survival time was 25.4 days $(\mathrm{DT}=27.4 ; \mathrm{SPC}=26.8 ; p=0.453)$.

\section{Demoralization Syndrome}

As previously reported, the prevalence of DS was $52.5 \%$ (Julião et al., 2016). No statistically significant differences were found between the two study groups at baseline with respect to prevalence of $\operatorname{DS}(p=$ 0.901). Within the DT group, DS shifted from $53.9 \%$ 
Table 2. Summary characteristics of participants at baseline $(N=80)$

\begin{tabular}{|c|c|c|c|}
\hline & Control group $(n=39)$ & DT group $(n=41)$ & Value of $p^{* *}$ \\
\hline \multicolumn{4}{|l|}{ Gender, $n(\%)$} \\
\hline Male & $18(43.9)$ & $19(48.7)$ & \multirow{2}{*}{0.823} \\
\hline Female & $23(56.1)$ & $20(51.3)$ & \\
\hline \multicolumn{4}{|l|}{ Age, years } \\
\hline Mean $(S D)$ & $66.1(12.9)$, range $=28-90$ & & \multirow{3}{*}{1.000} \\
\hline$\leq 65$ & $21(51.2)$ & $20(51.3)$ & \\
\hline$>65$ & $20(48.8)$ & $19(48.7)$ & \\
\hline \multicolumn{4}{|l|}{ Race/ethnicity, $n(\%)$} \\
\hline Caucasian & $38(95.0)$ & $37(94.9)$ & \multirow[t]{2}{*}{1.000} \\
\hline African & $2(5.0)$ & $2(5.1)$ & \\
\hline \multicolumn{4}{|l|}{ Marital status, $n(\%)$} \\
\hline Single & $9(21.9)$ & $6(15.4)$ & \multirow[t]{4}{*}{0.719} \\
\hline Married/common law & $14(34.2)$ & $18(46.2)$ & \\
\hline Divorced/separated & $7(17.1)$ & $5(12.8)$ & \\
\hline Widowed & $11(26.8)$ & $10(25.6)$ & \\
\hline \multicolumn{4}{|l|}{ Education, $n(\%)$} \\
\hline $\begin{array}{l}\text { Knows how to read and write } \\
\text { (without instruction) }\end{array}$ & $2(4.9)$ & $2(5.1)$ & \multirow[t]{4}{*}{0.844} \\
\hline Primary school & $16(39.2)$ & $12(30.8)$ & \\
\hline High school & $12(29.3)$ & $15(38.5)$ & \\
\hline Licensure & $11(26.8)$ & $10(25.6)$ & \\
\hline \multicolumn{4}{|l|}{ Religion, $n(\%)$} \\
\hline Catholic & $35(85.4)$ & $33(84.6)$ & \multirow[t]{2}{*}{1.000} \\
\hline Other & $6(14.6)$ & $6(15.4)$ & \\
\hline \multicolumn{4}{|l|}{ Diagnosis, $n(\%)$} \\
\hline Cancer* & $37(94.9)$ & $37(90.2)$ & \multirow[t]{2}{*}{0.676} \\
\hline Noncancer $^{\dagger}$ & $2(5.1)$ & $4(9.8)$ & \\
\hline \multicolumn{4}{|l|}{ Time since diagnosis, ${ }^{\dagger} n(\%)$} \\
\hline 1 year & $2(4.9)$ & $4(10.3)$ & \multirow[t]{3}{*}{0.103} \\
\hline $1-2$ years & $13(31.7)$ & $5(12.8)$ & \\
\hline$\geq 2$ years & $26(63.4)$ & $30(76.9)$ & \\
\hline \multicolumn{4}{|l|}{ Psychiatric drugs, $n(\%)$} \\
\hline Antidepressants & $22(56.4)$ & $24(58.5)$ & 1.000 \\
\hline Anxiolytics & $24(61.5)$ & $27(65.9)$ & 1.000 \\
\hline Neuroleptics & $6(15.4)$ & $1(2.4)$ & 0.520 \\
\hline Anticonvulsants & $17(41.5)$ & $22(56.4)$ & 0.263 \\
\hline \multicolumn{4}{|l|}{ Palliative Performance Scale ${ }^{\S}$} \\
\hline & $57.1(17.5)$, range $=30-90$ & & \\
\hline Mean $(S D)$ & $57.6(18.2)$ & $56.7(16.9)$ & 0.821 \\
\hline \multicolumn{4}{|l|}{ Mini-Mental State } \\
\hline & $24.4(1.9)$, range $=20-28$ & & \\
\hline Mean $(S D)$ & $24.1(2.0)$ & $24.7(1.9)$ & 0.212 \\
\hline
\end{tabular}

$\mathrm{DT}=$ dignity therapy; $S D=$ standard deviation.

$*$ Lung $(n=10)$, ovarian $(n=5)$, breast $(n=4)$, glioblastoma $(n=4)$, uterus $(n=4)$; tongue $(n=3)$, bladder $(n=2)$; caecum $(n=2)$, endometrium $(n=2)$, esophagus $(n=2)$, larynx $(n=2)$, stomach $(n=2)$, prostate $(n=2)$ unknown primary cancer, $(n=2)$, chronic myeloid leukemia $(n=1)$, dorsal neurinoma $(n=1)$, glioma $(n=1)$, melanoma $(n=1)$, neoplasm of the nose $(n=1)$, pancreas $(n=1)$, small bowel neuroendocrine tumor $(n=1)$, vascular arterial cancer $(n=1)$.

Lateral amyotrophic sclerosis $(n=2)$, trigeminal neuralgia $(n=1)$.

$\$$ Based on medical records or patient information.

$\S$ Palliative Performance Scale scores: $100 \%=$ healthy, $0 \%=$ dead.

** Fisher's exact test, with the exception of the Palliative Performance Status and the Mini-Mental State, where the Mann-Whitney test was used.

at baseline to $12.1 \%$ post-intervention $(p=0.002)$, while in the SPC group it shifted from 51.2 to $60.0 \%(p=0.343)$ (Table 3). Of the participants in the DT group who had DS at baseline, $82.4 \%$ no longer met the criteria for DS post-intervention. Of the
DT participants who did not have DS at baseline, $6.2 \%$ did so post-intervention $(p=0.008)$. Within the control group, of the participants who had DS at baseline, $18.6 \%$ no longer met the criteria for DS post-intervention. Of those in the SPC group who 
Table 3. Efficacy of DT with respect to demoralization syndrome and desire for death, within and between study groups

\begin{tabular}{lllll}
\hline \hline & & Baseline $(\%)$ & Post-intervention (\%) & Value of $p^{* *}$ \\
\hline Demoralization syndrome & DT group & 53.9 & 12.1 & 0.002 (within effect) \\
& $p$ value* & 0.901 (between effect) & $<0.001$ (between effect) & 0.343 (within effect) \\
Desire for death & Control group & 51.2 & 60.0 & 0.023 (within effect) \\
& DT group & 23.0 & 0 & \\
& $p$ value* & 0.582 (between effect) & 0.054 (between effect) & 1.0 (within effect)
\end{tabular}

$\mathrm{DT}=$ dignity therapy.

* Fisher's exact test.

** McNemar's exact test.

did not have DS at baseline, $38.8 \%$ met the criteria for DS post-intervention ( $p=0.205$ ).

DT was associated with a significant decrease in the incidence of DS compared to SPC (DT DS prevalence $=12.1 \%$; SPC DS prevalence $=60.0 \%$; $p<0.001$ ) (Table 3).

\section{Desire for Death}

The prevalence of DfD (DDRS $\geq 4$ ) for the entire cohort was $20.0 \%$ (Julião et al., 2013b). There were no statistically significant differences between the two study groups at baseline with respect to DfD ( $p=$ 0.582 ).

Within the DT group, the prevalence of DfD shifted from $23.0 \%$ at baseline to $0 \%$ post-intervention $(p=0.023)$. On the other hand, the prevalence of DfD in the SPC group shifted from $17.0 \%$ at baseline to $14.3 \%$ post-intervention ( $p=1.0$ ) (Table 3 ). Of those patients in the DT group with a DfD at baseline, none had a DfD post-intervention. On the other hand, no patient shifted from not having a DfD at baseline to a significant DfD post-DT $(p=0.015)$. Within the control group, of the participants who had a DfD at baseline, $66.7 \%$ had no desire for death upon follow-up; and $10.3 \%$ moved from not having a DfD at baseline to having a desire for death at follow-up $(p=1.0)$.

DT was associated with a significant decrease in DfD prevalence (DT DfD prevalence $=0 \%$; SPC DfD prevalence $=14.3 \% ; p=0.054)($ Table 3$)$.

\section{Sense of Dignity}

Of the 25 PDI questions, only 9 had a mean score $<3$, meaning that these patients did not identify that issue as being problematic (Chochinov et al., 2008). Most responses to the PDI questions at baseline (64\%) had an average score $\geq 3$, revealing intense dignity-related suffering (Chochinov et al., 2008).

Patients allocated to the DT group showed a statistically significant decrease in all PDI questions ex- cept for cognitive capacity, meaningful life, and healthcare support. Patients allocated to the control group showed a statistically significant decrease in only five PDI questions, particularly related to bodily functions/independence, physical symptoms, anxiety, sense of worth/value, and healthcare support. Compared with participants allocated to the control group, those who received DT showed a statistically significant reduction on 19 of 25 PDI items (with the exception of items 4, 9, 15, 21, 22, and 25 [Table 4]).

\section{DISCUSSION}

To the best of our knowledge, this is the first RCT to study the efficacy of DT for demoralization and DfD in terminally ill patients. It is also the first Portuguese study to look at the effect of this brief psychotherapeutic intervention on sense of dignity and sources of dignity-related distress. As previously reported by our research group, the patients in this RCT had high levels of psychosocial distress at baseline-namely, depression and anxiety (Julião et al., 2013a; 2014). This cohort of patients also experienced high levels of $\mathrm{DS}, \mathrm{DfD}$, and dignity-related distress.

DT appeared to significantly decrease DS, DfD, and SoD—that is, patients who received DT were significantly more likely than SPC patients to experience significant reductions in DS and DfD, along with improvement of their SoD. These results indicate that DT is an effective psychotherapeutic intervention for those dimensions of the end-of-life experience.

Many underlying causes could explain the mechanisms by which the DT reduces patient distress. We think that patients who engage in DT are better able to move beyond their sadness and vital suffering, to an activity imbued with meaning and purpose. In addition, the end-of-life period also offers opportunities for personal growth and deepening of relationships, and this might also be an important source of DT's efficacy. 
Table 4. Differences in PDI item scores between T1 and T2, comparing DT and SPC

\begin{tabular}{|c|c|c|c|c|c|}
\hline \multirow{2}{*}{ PDI questions } & \multicolumn{2}{|c|}{ DT group } & \multirow[b]{2}{*}{$\begin{array}{c}p \\
\text { value }^{*}\end{array}$} & \multicolumn{2}{|c|}{ Control group } \\
\hline & Mean $(S D)$ & Median & & Mean $(S D)$ & Median \\
\hline $\begin{array}{l}\text { 1. Not being able to carry out tasks associated with daily } \\
\text { living }\end{array}$ & $-0.70(1.16)$ & -1.00 & 0.002 & $0.26(1.29)$ & 0.00 \\
\hline $\begin{array}{l}\text { 2. Not being able to attend to my bodily functions } \\
\text { independently }\end{array}$ & $-0.73(1.18)$ & 0.00 & 0.000 & $0.41(1.10)$ & 0.00 \\
\hline 3. Experiencing physically distressing symptoms & $-1.58(1.60)$ & -2.00 & 0.006 & $-0.53(1.33)$ & 0.00 \\
\hline 4. Feeling that how I look to others has changed significantly & $-0.88(1.22)$ & -1.00 & 0.127 & $-0.24(1.50)$ & 0.00 \\
\hline 5. Feeling depressed & $-1.09(1.49)$ & -1.00 & 0.000 & $0.09(1.03)$ & 0.00 \\
\hline 6. Feeling anxious & $-0.94(1.32)$ & -1.00 & 0.000 & $0.33(.85)$ & 0.00 \\
\hline 7. Feeling uncertain about my illness and treatment & $-1.24(1.20)$ & -1.00 & 0.000 & $-0.03(1.11)$ & 0.00 \\
\hline 8. Worrying about my future & $-1.48(1.35)$ & -2.00 & 0.000 & $0.12(0.81)$ & 0.00 \\
\hline 9. Not being able to think clearly & $-0.15(1.25)$ & 0.00 & 0.070 & $0.35(0.95)$ & 0.00 \\
\hline 10. Not being able to continue with my usual routines & $-0.85(1.28)$ & -1.00 & 0.004 & $-0.03(0.90)$ & 0.00 \\
\hline 11. Feeling like I am no longer who I was & $-1.82(1.40)$ & -2.00 & 0.000 & $-0.15(1.28)$ & 0.00 \\
\hline 12. Not feeling worthwhile or valued & $-1.30(1.76)$ & -1.00 & 0.014 & $-0.38(1.07)$ & 0.00 \\
\hline 13. Not being able to carry out important roles & $-1.18(1.51)$ & -1.00 & 0.021 & $-0.29(1.45)$ & 0.00 \\
\hline 14. Feeling that life no longer has meaning or purpose & $-1.73(1.55)$ & -2.00 & 0.000 & $0.00(1.41)$ & 0.00 \\
\hline $\begin{array}{l}\text { 15. Feeling that I have not made a meaningful and lasting } \\
\text { contribution during my lifetime }\end{array}$ & $-0.21(1.47)$ & 0.00 & 0.524 & $0.06(1.01)$ & 0.00 \\
\hline 16. Feeling I have 'unfinished business & $-1.27(1.59)$ & -1.00 & 0.011 & $-0.32(1.32)$ & 0.00 \\
\hline 17. Concern that my spiritual life is not meaningful & $-0.78(1.29)$ & 0.00 & 0.021 & $-0.12(0.77)$ & 0.00 \\
\hline 18. Feeling that I am a burden to others & $-1.03(1.21)$ & -1.00 & 0.002 & $-0.15(1.16)$ & 0.00 \\
\hline 19. Feeling that I don't have control over my life & $-1.21(1.39)$ & -1.00 & 0.000 & $0.06(1.04)$ & 0.00 \\
\hline $\begin{array}{l}\text { 20. Feeling that my illness and care needs have reduced my } \\
\text { privacy }\end{array}$ & $-1.03(1.31)$ & -1.00 & 0.001 & $0.03(0.97)$ & 0.00 \\
\hline $\begin{array}{l}\text { 21. Not feeling supported by my community of friends and } \\
\text { family }\end{array}$ & $-0.56(1.32)$ & 0.00 & 0.249 & $-0.12(0.78)$ & 0.00 \\
\hline 22. Not feeling supported by my healthcare providers & $-0.12(0.78)$ & 0.00 & 0.175 & $-0.26(0.79)$ & 0.00 \\
\hline $\begin{array}{l}\text { 23. Feeling like I am no longer able to mentally "fight" the } \\
\text { challenges of my illness }\end{array}$ & $-1.30(1.40)$ & -1.00 & 0.000 & $0.15(0.99)$ & 0.00 \\
\hline 24. Not being able to accept the way things are & $-1.52(1.42)$ & -1.00 & 0.000 & $-0.03(0.97)$ & 0.00 \\
\hline 25. Not being treated with respect or understanding by others & $-0.58(1.52)$ & 0.00 & 0.206 & $-0.15(0.61)$ & 0.00 \\
\hline
\end{tabular}

$\mathrm{DT}=$ dignity therapy; $S D=$ standard deviation; $\mathrm{SPC}=$ standard palliative care.

Bold $=$ significant values.

* Mann-Whitney test.

According to de Figueiredo (1993), the key elements of DS include marked incompetence, a profound inability to express feelings and develop tasks properly when confronted with illness, leading to a state of inability, uncertainty, profound lethargy, and doubts concerning the future. According to the author, demoralized patients lose their cognitive mapping, plunging into vital incompetence. Perhaps DT influences DS by calling upon patients to actively engage in the retelling of their stories and the creation of a legacy document. This task requires that they not only summarize certain aspects of their life, but that they do so in the service of those they are about to leave behind. This future orientation of DT sees demoralized patients, who have lost hope in the future, having to invest in others who will outlive them so that they can take their words into a future the patients will no longer be a part of. Thus, DT encourages demoralized and emotionally lethargic patients to revive, to regain some of their inner strength in order to engage in and enable the creation of their generativity document. It demands a future orientation, which sees participants achieve mastery, by way of creating a tangible product that takes them beyond their present circumstances and thus reduces their self-perception of incompetence. As one patient put it, "Yes, I can achieve this. It's possible, and I can do it and believe in something more than my empty and disease-full present."

DT was effective in reducing a desire for death, both within the intervention group $(p=0.023)$ as well as compared to the control group $(p=0.054)$. These results suggest that DT might reduce requests for a hastened death in patients approaching the end of their lives. Dignity therapy was able to eliminate DfD in every participant in the DT group who had originally scored above the critical DfD threshold criterion separating them from those without a desire to 
die. Patients allocated to the control group showed no significant change in their desire to die sooner $(p=$ 1.0), which means that the multidisciplinary intervention was not able to reduce their DfD.

Again, there are many possible explanations for how DT might affect a patient's DfD. By focusing on the personal narrative and experiencing a therapeutic encounter that includes active listening, DT may reinforce a sense of meaning, of being valued, and of being important, despite the challenges caused by one's illness, therefore helping patients to relinquish a death-hastening stance. As reported elsewhere (Chochinov et al., 1995; Julião et al., 2013a), DfD is fluctuant and can be influenced by a therapeutic stance comprised of compassion and unwavering support in the face of suffering.

Aside from higher levels of anxiety and depression, the participants in our study also experienced more pronounced dignity-related distress compared with those reported in the prior DT RCT (Chochinov et al., 2011). Only 9 of 25 PDI items had a mean score $<3$, indicating an absence of significant dignity-related distress. The associations among depression, anxiety, and dignity-related distress have been welldocumented (Chochinov et al., 2011; Julião, 2014). Based on our findings, DT appears to be an effective way to bolster dignity by way of decreasing dignityrelated distress. DT outperformed SPC in mitigating suffering based on "dependency" (PDI items 1, 2, 10, 18 , and 20), "existential suffering" $(4,8,11,12,14,15$, 16,17 , and 24), and "control" (13, 19, and 23).

A factor analysis performed by Hack et al. (2010) within a cohort of patients with end-stage cancer showed that hopelessness, privacy boundaries, and intimate daily hygiene activities (including diaper changes and assisted baths) were most highly associated with SoD. This offers guidance to caregivers and health professionals in pursuit of providing dignityconserving care for patients nearing death (Chochinov, 2002). Dignity therapy is anchored in the essential premise of respect. Within the very medically oriented world, DT therapists create private and hopeful moments wherein patients can feel valued and return to looking at the world and those who provide them care as being imbued with humanity, with compassion, thus enhancing their experience of care, despite the challenges of relinquishing privacy and accepting the intimate dependencies of care. DT allows patients to reclaim a sense of control, even if for a brief period, as they approach death. As one patient put it,

I thought life had reached an end without yet having ended. I belonged to a strange sensation that I didn't have any control over my daily activities. [The] world's adversity took control over me. This disease gave me the false idea that it ruled me. It's not true. I can't do my hygiene and other regular tasks anymore, but I'm capable of producing incredible things-like the legacy document-far beyond my ill body. I can create beyond my boundaries [and] dependencies, and I discovered that dignity therapy is a way to achieve that.

\section{LIMITATIONS OF THE STUDY}

Like any study, ours had its limitations. Our sample consisted primarily of older patients. As such, the influence of DT within younger cohorts remains uncertain and deserves further examination. Despite a relatively small sample size, we were able to detect significant differences between and within groups, indicating clinically important benefits in terms of the psychosocial dimensions of end-of-life suffering. The majority of our participants had end-stage malignancies. Our future research will aim to explore the utility of DT in other terminal conditions, such as end-stage organ failure. In the present trial, DT was performed exclusively by one therapist (M.J.), who carefully followed the DT protocol (Chochinov et al., 2005; Chochinov, 2011) and was trained by the originators of this therapeutic approach. He was not and could not be blinded to study arm assignment, which raises the question of potential bias. However, patients in both groups received equalquality palliative care (SPC), and all the evaluations, treatment plans, and direct interventions were carried out by the entire multidisciplinary team.

It is important to place the findings of our study within the context of Portuguese culture and religion, especially with respect to views regarding suffering, death, and dying. Most Portuguese are Catholics and accept the end of life and its related suffering as beyond question and in the "hands of God." In Portugal, particularly among the elderly, there has traditionally been great reticence to express feelings regarding physical and psychological discomfort. Consequently, healthcare providers frequently avoid engaging in conversations pertaining to the topics of death and dying. While this might present a challenge to implementing DT, it is notable that most patients approached to participate in our study did so (only 4 out of the 92 patients assessed for eligibility declined to participate), and they benefited from the opportunity to share their reflections, hopes, and wishes for the loved ones they were about to leave behind. This could affect the way people are, or are not, accepting of death, and perhaps even the way that death anxiety plays itself out in Portuguese society. Again, having a therapist who offers an intervention based on life's positive aspects, achievements, human relationships, and wishes that will be collected 
in a legacy document might give terminally ill people a chance to reconnect with something more simple and pure, higher and more meaningful, that speaks for love, caring for others, and not for guilt or punishment, thus alleviating some of the ancient and heavy-laden Catholic view of death.

Another disadvantage that must be acknowledged is that assessments were performed at baseline and only four days after the intervention. No conclusions can therefore be drawn about the time stability of the variables.

DT provided beneficial effects in the psychological dimensions of the distress experienced by patients near the end of life. While further research on DT is warranted, mounting evidence suggests that this unique psychotherapeutic approach is an important tool that should be included in the clinical care of terminally ill patients and their families.

\section{STATEMENT OF AUTHORSHIP}

M.J. and A.B. were responsible for study conception and design. M.J. was also responsible for supervising the study, analyzing the data, and writing the initial draft and final report. F.O. was responsible for scale assessments. B.N. supervised statistical analysis of the data. A.V.C. helped with revising the final report. All of the coauthors helped to revise the final manuscript and had full access to all of the data.

\section{CONFLICTS OF INTERESTS}

The authors hereby state that they have no conflicts of interest to declare.

\section{ACKNOWLEDGMENTS}

A special word of gratitude to Professor Chochinov for his unwavering guidance and wisdom. And to each and every patient who participated in our study: without their commitment and generosity, this study would not have been possible.

\section{REFERENCES}

Block, S.D. (2000). Assessing and managing depression in the terminally ill patient. ACP-ASIM End-of-Life Care Consensus Panel. American College of Physicians-American Society of Internal Medicine. Annals of Internal Medicine, 132(2), 209-218.

Block, S.D. (2001). Perspectives on care at the close of life. Psychological considerations, growth, and transcendence at the end of life: The art of the possible. The Journal of the American Medical Association, 285(22), 2898-2905.

Breitbart, W., Rosenfeld, B., Pessin, H., et al. (2000). Depression, hopelessness, and desire for hastened death in terminally ill patients with cancer. The Journal of the American Medical Association, 284(22), 2907-2911.

Chochinov, H.M. (2002). Dignity-conserving care: A new model for palliative care. Helping the patient feel valued. The Journal of the American Medical Association, 287(17), 2253-2260.

Chochinov, H.M. (2007). Dignity and the essence of medicine: The A, B, C, and D of dignity-conserving care. British Medical Journal, 335, 184-187.

Chochinov, H.M. (2011). Dignity therapy: Final words for final days. New York: Oxford University Press.

Chochinov, H.M., Wilson, K.G., Enns, M., et al. (1995). Desire for death in the terminally ill. The American Journal of Psychiatry, 152(8), 1185-1191.

Chochinov, H.M., Hack, T., McClement, S., et al. (2002). Dignity in the terminally ill: A developing empirical model. Social Science \& Medicine, 54(3), 433-443.

Chochinov, H.M., Hack, T., Hassard, T., et al. (2005). Dignity therapy: A novel psychotherapeutic intervention for patients near the end of life. Journal of Clinical Oncology, 23(24), 5520-5525. Available from http://ascopubs.org/doi/pdf/10.1200/JCO.2005.08.391.

Chochinov, H.M., Hassard, T., McClement, S., et al. (2008). The Patient Dignity Inventory: A novel way of measuring dignity-related distress in palliative care. Journal of Pain and Symptom Management, 36(6), 559-371. Epub ahead of print Jun 24. Available from http://www. jpsmjournal.com/article/S0885-3924(08)00250-9/pdf.

Chochinov, H.M., Kristjanson, L.J., Breitbart, W., et al. (2011). The effect of dignity therapy on distress and end-of-life experience in terminally ill patients: A randomised controlled trial. The Lancet. Oncology, 12(8), 753-762. Epub ahead of print Jul 6. Available from https:/www.ncbi.nlm.nih.gov/pmc/articles/PMC3185066/ pdf/nihms-315318.pdf.

de Figueiredo, J.M. (1993). Depression and demoralization: Phenomenologic differences and research perspectives. Comprehensive Psychiatry, 34(5), 308-11.

Hack, T.F., McClement, S.E., Chochinov, H.M., et al. (2010). Learning from dying patients during their final days: Life reflections gleaned from dignity therapy. Palliative Medicine, 24(7), 715-723. Epub ahead of print Jul 6. Available from http://journals.sagepub.com/doi/ pdf/10.1177/0269216310373164.

Hall, S., Goddard, C., Opio, D., et al. (2011). A novel approach to enhancing hope in patients with advanced cancer: A randomised phase II trial of dignity therapy. BMJ Supportive \& Palliative Care, 1(3), 315-321. Epub ahead of print Oct 9.

Hudson, P.L., Kristjanson, L.J., Ashby, M., et al. (2006). Desire for hastened death in patients with advanced disease and the evidence base of clinical guidelines: A systematic review. Palliative Medicine, 20(7), 693-701.

Julião, M. (2014). Eficácia da terapia da dignidade na patologia psicossocial de doentes seguidos em Cuidados paliativos: Ensaio clínico aleatorizado e controlado [in Portuguese]. Doctoral dissertation. Lisbon: Faculdade de Medicina da Universidade de Lisboa.

Julião, M. (2015). Human dignitas, dignity in care: A precious need, International Journal of Emergency Mental Health and Human Resilience, 17(3), 598-599. Available from https://www.omicsonline.com/open-access/ human-dignitas-dignity-in-care-a-precious-need-15224821-1000e225.pdf.

Julião, M., Barbosa, A., Oliveira, F., et al. (2013a). Efficacy of dignity therapy for depression and anxiety in terminally ill patients: Early results of a randomized 
controlled trial. Palliative \& Supportive Care, 11(6), 481-489. Epub ahead of print Mar 19.

Julião, M., Barbosa, A., Oliveira, F., et al. (2013b). Prevalence and factors associated with desire for death in patients with advanced disease: Results from a Portuguese cross-sectional study. Psychosomatics, 54(5), 451-457. Epub ahead of print Mar 7.

Julião, M., Oliveira, F., Nunes, B., et al. (2014). Efficacy of dignity therapy on depression and anxiety in Portuguese terminally ill patients: A phase II randomized controlled trial. Journal of Palliative Medicine, 17(6), 688-695. Epub ahead of print Apr 15.
Julião, M., Nunes, B. \& Barbosa, A. (2016). Prevalence and factors associated with demoralization syndrome in patients with advanced disease: Results from a crosssectional Portuguese study. Palliative \& Supportive Care, 14(5), 468-473. Epub ahead of print Jan 6.

Kissane, D.W., Clarke, D.M. \& Street, A.F. (2001). Demoralization syndrome: A relevant psychiatric diagnosis for palliative care. Journal of Palliative Care, 17(1), $12-21$.

Mystakidou, K., Rosenfeld, B., Parpa, E., et al. (2005). Desire for death near the end of life: The role of depression, anxiety, and pain. General Hospital Psychiatry, 27(4), 258-262. 